Pacific Journal of Mathematics

RINGS OF FUNCTIONS DETERMINED BY ZERO-SETS 


\title{
RINGS OF FUNCTIONS DETERMINED BY ZERO-SETS
}

\section{HUGH GORDON}

\begin{abstract}
Pseudocompactness and realcompactness can be defined in a more natural and more general setting than the usual one. One part of what is done here is simply to point out that much of the theory of rings of continuous functions applies without essential change in more general circumstances. The discussion includes, for example, analogues of $\beta X, \nu X, z$-ultrafilters, $C(X)$ and $C^{*}(X)$, but all for a zero-set space, instead of for a topological space.

There is another respect, besides greater generality, in which the theory of zero-set spaces differs from that of topological spaces. Using the definitions of subspace and product space which are obvious and natural for zero-set spaces, this paper obtains, for such spaces, a number of results which are known to fail for topological spaces. Most notably, a product of any number of pseudocompact zero-set spaces is pseudocompact, even though the product of just two pseudocompact topological spaces may fail to be pseudocompact. Also a countable union of realcompact subspaces of a zero-set space is realcompact; again the corresponding statement does not hold even for two topological subspaces.
\end{abstract}

One approach to generalizing the concepts of pseudocompactness and realcompactness has been made by Lorch [5]. What we do here is, relying heavily on the work of Lorch, to go well beyond that work by emphasizing particularly a special case, which is general enough to include the important applications in analysis.

Let us set forth the matters of the preceding paragraph in greater detail. Lorch starts with a uniformly closed ring of bounded functions on a set $X$; here as elsewhere, we understand "function" to mean "real-valued function." Given such a ring $\mathscr{R}$, we may consider the collection $\mathscr{Z}$ of all zero-sets of functions in $\mathscr{R}$; i.e., the collection of all sets of the form $\{x \in X \mid f(x)=0\}$ for all $f \in \mathscr{R}$. Note that there can be a number of different rings $\mathscr{R}$ having the same collection $\mathscr{F}$ of zero-sets. But whether $X$ is pseudocompact, with respect to some $\mathscr{R}$, in Lorch's theory turns out to depend only on $\mathscr{Z}$, not on $\mathscr{R}$; the same holds for realcompact. Among the rings $\mathscr{R}$ corresponding to any $\mathscr{z}$ there is a largest one-and we depart from the work of Lorch by fixing our attention on this largest one. This largest ring consists of all bounded functions $f$ such that $f^{-1} F \in \mathscr{Z}$ for every closed set $F$ of real numbers, as will become clear in $\S 2$ below. Note that rings of continuous functions are the largest rings 
for their collections of zero-sets. But rings of Baire functions and rings of measurable functions also have this property. In this paper we begin with some $\mathscr{Z}$ and study especially the largest ring $\mathscr{R}$ which corresponds.

In the theory of Lorch, the ring $\mathscr{R}$ is a generalization of the ring $C^{*}(X)$ of bounded continuous functions. But many theorems about rings of continuous functions have reference to the ring $C(X)$ of all continuous functions. It is natural then to seek a generalization for $C(X)$ corresponding to $\mathscr{R}$ for $C^{*}(X)$. While serious difficulties arise in the general case, we shall have no trouble in the case where $\mathscr{R}$ has the maximality property of the preceding paragraph. Theorem 3.7 shows that three obvious definitions for the analogue of $C(X)$ coincide. This analogue appears in many of the theorems below; for example, $X$ is pseudocompact if and only if the analogues of $C(X)$ and $C^{*}(X)$ coincide.

We shall begin the development of our theory by giving the definition of a zero-set structure. By a zero-set structure on a set $X$ we shall mean a collection $z$ of subsets of $X$ satisfying certain set-theoretic axioms. These axioms are equivalent to the assertion that $\mathscr{z}$ is the collection of zero-sets of a uniformly closed ring of bounded functions. Recalling that proximity structures are in a natural sense intermediate between topologies and uniform structures, one is led to ask where zero-set structures fit in. The answer, which becomes apparent in $\S 3$ and $\S 4$ below, is that zero-set structures fall between topologies and proximity structures; additional details about this point will be found in the body of the paper.

\section{Notation and terminology.}

1.1. We shall use the word "function" only in reference to a real-valued function. Given a set $X$, the totality of functions on $X$ forms a ring with the operations specified by:

$$
\begin{aligned}
(f+g)(x) & =f(x)+g(x) \\
(f g)(x) & =f(x) g(x)
\end{aligned}
$$

and a lattice with the operations specified by:

$$
\begin{aligned}
& (f \vee g)(x)=\max (f(x), g(x)) \\
& (f \wedge g)(x)=\min (f(x), g(x)) .
\end{aligned}
$$

Whenever we speak of a ring or a lattice of functions on $X$, we mean always a subring or a sublattice of the ring or lattice of all functions just mentioned. The constant function whose value at each 
point is $\alpha$ will also be denoted by $\alpha$. It is well-known that if a uniformly closed ring of bounded functions on a set $X$ contains the constants, then it is a lattice of functions, in the sense just specified. Thus in particular, if it contains some $f$, it also contains $f^{+}, f^{-}$and $|f|$ defined by

$$
f^{+}=f \vee 0, f^{-}=(-f)^{+}
$$

and $|f|=f^{+}+f^{-}$.

1.2. $f^{-1}$ refers always to the inverse image; the symbol $1 / f$ is used for the function defined by

$$
(1 / f)(x)=1 / f(x) \text {. }
$$

If $f: X \rightarrow \boldsymbol{R}$, where of course $\boldsymbol{R}$ is the real numbers, then $\boldsymbol{Z} f$ denotes $f^{-1}(0)$ and is called the zero-set of $f$.

1.3. All topologies which appear below are completely regular, and we understand completely regular to imply Hausdorff.

Whenever we refer to a topology, proximity structure or uniform structure on $\boldsymbol{R}$, without further specification, we mean of course the standard structure of the kind mentioned.

2. Definition of a zero-set space. We begin by giving the abstract definition of a zero-set structure. Theorems 2.3 and 3.5 will subsequently serve to explain the relationship to rings of functions and to justify the use of the word "zero-set" in the definition.

DEFINITION 2.1. Let $X$ be a set and $\mathscr{Z}$ be a collection of subsets of $X$. Suppose:

(1) For each pair of distinct points of $X$, there is a $Z \in \mathscr{F}$ which contains just one of the points.

(2) $\mathscr{c}$ is closed under finite union; in particular, $\phi \in \mathscr{L}$.

(3) $\mathscr{z}$ is closed under countable intersection; in particular, $X \in \check{z}$.

(4) Whenever $A$ and $B \in \mathscr{Z}$ and $A \cap B=\phi$, then there are $C$ and $D \in \mathscr{Z}$ with $A \subset X \backslash C, B \subset X \backslash D$ and $(X \backslash C) \cap(X \backslash D)=\dot{\phi}$.

(5) Whenever $Z \in \mathscr{L}$, there are $Z_{1}, Z_{2}, \cdots \in \mathscr{K}$ such that:

$$
X \backslash Z=\bigcup_{n=1}^{\infty} Z_{n} \text {. }
$$

Then we call $\mathscr{Z}$ a zero-set structure on $X$ and call $(X, \mathscr{Z})$ a zero-set space. We usually abbreviate by writing $X$ for $(X, \mathscr{L})$.

2.2. The totality of closed sets of a $T_{0}$ topological space satisfies 
(1), (2) and (3) above. If the space is normal, (4) is also satisfied, but (5) need not be. On the other hand, the closed sets of the space $\Gamma$ of [2, Exercise $3 \mathrm{~K}]$ satisfy (5) but not (4).

TheOREM 2.3. Let $X$ be a set and $/ l$ be a uniformly closed ring of bounded functions on $X$. Suppose $\mathscr{C l}$ separates points and contains the constants. Then

$$
\mathscr{Z}=\{\boldsymbol{Z} f \mid f \in \mathscr{C}\}
$$

is a zero-set structure on $X$.

Proof. Certainly (1) of 2.1 is satisfied. Since $\phi \in \mathscr{Z}$ and $Z(f g)=$ $Z f \cup Z g,(2)$ is satisfied. Since $X \in \mathscr{Z}$ and

$$
\boldsymbol{Z} \sum_{n=1}^{\infty}\left(\left|f_{n}\right| \wedge 2^{-n}\right)=\bigcap_{n=1}^{\infty} \boldsymbol{Z} f_{n},
$$

(3) is satisfied. If $A=\boldsymbol{Z} f$ and $B=\boldsymbol{Z} g$ are disjoint, then the sets

$$
C=\boldsymbol{Z}(|g|-|f|)^{+} \text {and } D=\boldsymbol{Z}(|f|-|g|)^{+}
$$

serve to verify (4). To verify (5) for $Z=Z f$, define

$$
Z_{n}=Z[|f|-(1 / n)]^{-} \text {. }
$$

2.4. The following two lemmas will be needed later.

Lemma. Let $\mathscr{B}$ be a collection of subsets of $X$ closed under finite union and countable intersection. Let $\mathscr{I l}$ be the set of all functions $f$ with the property that $f^{-1} F \in \mathscr{B}$ for each closed set $F$ of $\mathbf{R}$. Then $\mathscr{C l}$ is a uniformly closed ring.

Proof. Let $\mathscr{C}=\{X \backslash A \mid A \in \mathscr{B}\}$; then $\mathscr{C}$ is closed under finite intersection and countable union. Also $\mathscr{C l}$ is the set of functions $f$ such that $f^{-1} U \in \mathscr{C}$ for every $U$ open in $\boldsymbol{R}$. Let $f_{1}$ and $f_{2} \in \mathscr{C l}$ be given. Define $g: X \rightarrow \boldsymbol{R} \times \boldsymbol{R}$ by

$$
g(x)=\left(f_{1}(x), f_{2}(x)\right) .
$$

Each open set of $\boldsymbol{R} \times \boldsymbol{R}$ is the union of countably many sets of the form $V_{1} \times V_{2}$ where $V_{1}$ and $V_{2}$ are open in $\boldsymbol{R}$. Since for each choice of $V_{1} \times V_{2}$ we have

$$
g^{-1}\left(V_{1} \times V_{2}\right)=f_{1}^{-1} V_{1} \cap f_{2}^{-1} V_{2} \in \mathscr{C},
$$

$g$ has the property that $g^{-1} V \in \mathscr{C}$ for each open $V$ of $\boldsymbol{R} \times \boldsymbol{R}$. Since the mappings $\delta: \boldsymbol{R} \times \boldsymbol{R} \rightarrow \boldsymbol{R}$ and $\pi: \boldsymbol{R} \times \boldsymbol{R} \rightarrow \boldsymbol{R}$ defined by $\delta(\alpha, \beta)=$ 
$\alpha-\beta$ and $\pi(\alpha, \beta)=\alpha \beta$ are continuous, $\delta \circ g$ and $\pi \circ g$ have the property that the inverse image of an open set lies in $\mathscr{C}$. In other words, $f_{1}-f_{2}=\delta \circ g$ and $f_{1} f_{2}=\pi \circ g$ belong to $\mathscr{C l}$. In short $\mathscr{C}$ is a ring.

It remains to show that $\mathscr{C}$ is uniformly closed. Let $f_{1}, f_{2}, \ldots \in \mathscr{C l}$ and let $f_{n} \rightarrow f$ uniformly. It is enough to show $f^{-1}(\alpha, \beta) \in \mathscr{C}$ whenever $\alpha<\beta$. For each positive integer $k$, choose $n_{k}$ such that

$$
\left|f_{n_{k}}(x)-f(x)\right|<1 / k
$$

for all $x \in X$. Let

$$
U_{k}=f_{n_{k}}^{-1}(\alpha+1 / k, \beta-1 / k) .
$$

Note $U_{k} \in \mathscr{C}$ and $U_{k} \subset f^{-1}(\alpha, \beta)$ for all $k$. But each $x \in f^{-1}(\alpha, \beta)$ belongs to $U_{k}$ for some $k$. Thus

$$
f^{-1}(\alpha, \beta)=\cup U_{k} \in \mathscr{C}
$$

LEMma 2.5. Let $\mathscr{Z}_{0}$ be a collection of subsets of $X$ such that for each pair of points of $X$ there is a $Z \in \mathscr{Z}_{0}$ containing just one of them. Let of be the collection of all countable intersections of finite unions of sets in $\mathscr{Z}_{0}$. Suppose for each $Z \in \mathscr{Z}_{0}$ there is an $f$ such that both $\boldsymbol{Z} f=Z$ and $f^{-1} F \in \mathscr{Z}$ for every $F$ closed in $\boldsymbol{R}$. Then \& is a zero-set structure on $X$.

Proof. Note first that given any function $f$ on $X$ with the property that $f^{-1} F \in \mathscr{Z}$ for all $F$ closed in $\boldsymbol{R}$, then the function $|f| \wedge 1$ also has this property and

$$
Z f=Z(|f| \wedge 1)
$$

Thus, letting $\mathscr{C l}$ be the totality of bounded functions on $X$ with the property and letting $\mathscr{Z}^{\prime}$ be $\{\boldsymbol{Z} f \mid f \in \mathscr{C}\}$, we have $\mathscr{Z}_{0} \subset \mathscr{Z}^{\prime}$. By Lemma 2.4, $\mathscr{C}$ is a uniformly closed ring. Since we understand the definition of $\mathscr{Z}$ to imply $\phi$ and $X \in \mathscr{Z}$, $\mathscr{C}$ contains the constants. Also $\mathscr{C l}$ separates points. Thus $\mathscr{Z}^{\prime}$ is a zero-set structure on $X$. We complete the proof by showing $\mathscr{Z}=\mathscr{Z}^{\prime \prime}$; but, since $\mathscr{L}^{\prime} \subset \mathscr{Z}$ obviously, we need only show $\mathscr{Z} \subset \mathscr{Z}^{\prime}$. To this end, since $\mathscr{Z}^{\prime}$ is closed under countable intersection and finite union, it suffices to recall $\mathscr{Z}_{0} \subset \mathscr{Z}^{\prime}$.

DeFinition 2.6. Let $(X, \mathscr{Z})$ be a zero-set space. We call the sets in $\mathscr{Z}$ zero-sets and we call their complements, with respect to $X$, cozero-sets. 
2.7. That the following definition actually describes a completely regular topology will be apparent after Theorem 3.5 is proved.

Definition. Given a zero-set space $X$, we endow $X$ with a topology by taking the cozero-sets as a base (for the open sets). In other words, the zero-sets are a base for the closed sets.

2.8. Starting with a completely regular topological space, we can of course define a zero-set space by using the zero-sets of the continuous functions as the zero-sets of the structure. The topology defined, in turn, by this zero-set structure is the original topology. But, on the other hand, the same topology can arise from more than one zero-set structure. For example, the Baire sets on a metric space form a zero-set structure, which has for topology the discrete topology.

There are examples, however, of topological spaces which admit unique zero-set structures. A topological space with a countable base has this property. In fact, if the topology determined by a zero-set structure has a countable base, every open set is a cozero-set, for the given zero-set structure. To see this, we note that the cozerosets form a base for the topology and hence a countable subcollection of them is also a base for the topology; thus every open set is a countable union of cozero-sets, and hence is a cozero-set. Other examples of topological spaces which admit a unique zero-set structure are those topological spaces which admit unique uniform structures; this statement will become apparent in $\$ 4$.

\section{Zero-set functions.}

3.1. If $X$ and $Y$ are zero-set spaces, we call a mapping $\varphi: X \rightarrow Y$ a zero-set mapping if $\varphi^{-1} Z$ is a zero-set of $X$ for each zero-set $Z$ of $Y$. Equivalently, $\varphi$ is a zero-set mapping exactly when the inverse image of every cozero-set is a cozero-set. That the composition of two zero-set mappings is a zero-set mapping is almost too trivial to mention.

In case $Y=\boldsymbol{R}$, the cozero-sets of $Y$ are precisely the open sets of $Y$-since we obviously take this statement as a definition of the standard zero-set structure of $\boldsymbol{R}$. Each of these open sets is a countable union of open intervals. Thus $f: X \rightarrow \boldsymbol{R}$ is a zero-set mapping exactly when the inverse image of each open interval is a cozero-set.

3.2. We now endow each zero-set space $X$ with a proximity structure. By a proximity structure we mean a symmetric relation on the set of all subsets of $X$ satisfying the axioms stated below; 
Cech [1] calls such a structure a uniformizable proximity structure. Rather than describing those pairs of subsets which are to be proximal, we describe those which are to not be proximal, in other words, are to be distal. We define two subsets of $X$ to be distal if they are contained in disjoint zero-sets. We list and check the axioms for a proximity structure:

(1) $A$ and $B \cup C$ are proximal if and only if either $A$ and $B$ are proximal or $A$ and $C$ are proximal.

If $A$ and $B \cup C$ are distal, then $A$ and $B$ are obviously distal, as are $A$ and $C$. Conversely if $A \subset Z_{1}, A \subset Z_{2}, B \subset Z_{3}$ and $C \subset Z_{4}$ with $Z_{1} \cap Z_{3}=Z_{2} \cap Z_{4}=\phi$, then $A \subset Z_{1} \cap Z_{2}, B \cup C \subset Z_{3} \cup Z_{4}$, and $Z_{1} \cap Z_{2} \cap\left(Z_{3} \cup Z_{4}\right)=\phi$.

(2) $\{x\}$ and $\{y\}$ are proximal if and only if $x=y$.

"If" is obvious. If $x \neq y$, we may use (1) of 2.1 to find a zeroset $Z$ containing just one of $x$ and $y$. Say $x \in Z$ and $y \notin Z . \quad X \backslash Z$ is a union of zero-sets by (5) of 2.1; thus for some zero-set $Z^{\prime} \subset X \backslash Z$ we have $y \in Z^{\prime}$.

(3) No subset of $X$ is proximal to $\phi$.

Obvious.

(4) If $A$ and $B$ are distal, then there are $C$ and $D$ such that $X=C \cup D, A$ and $C$ are distal and $B$ and $D$ are distal.

Take disjoint zero-sets $Z_{1}$ and $Z_{2}$ containing $A$ and $B$ respectively. By (4) of 2.1 there are zero-sets $C$ and $D$ such that $Z_{1} \subset X \backslash C, Z_{2} \subset X \backslash D$ and $(X \backslash C) \cap(X \backslash D)=\phi$. This $C$ and $D$ are as required.

3.3. The following lemma will be used in proving the theorem which follows it and also in $\S 4$. We recall that a proximity mapping is a mapping $f$, from one proximity space to another, with the property that $f(A)$ and $f(B)$ are proximal whenever $A$ and $B$ are. An equivalent property is that $f^{-1}(C)$ and $f^{-1}(D)$ be distal whenever $C$ and $D$ are.

Lemma. Let $f: X \rightarrow \boldsymbol{R}$. Then $f$ is a zero-set mapping if and only if $f$ is a proximity mapping.

Proof. Suppose first that $f$ is a zero-set mapping. Let $A, B \subset X$ be such that $f A$ and $f B$ are distal in $R$. Then $\operatorname{cl}_{R} f A$ and $\operatorname{cl}_{R} f B$ are disjoint zero-sets of $\boldsymbol{R}$ and hence their inverse images under $f$ are disjoint zero-sets of $X$ containing $A$ and $B$ respectively. Thus $A$ and $B$ are distal.

Conversely suppose $f$ is a proximity mapping. Let $\alpha<\beta$ be real numbers. For each integer $n,\left[\alpha+n^{-1}, \beta-n^{-1}\right]$ and $\boldsymbol{R} \backslash(\alpha, \beta)$ are distal in $\boldsymbol{R}$ and hence their inverse images under $f$ are distal in $X$. Thus for each $n$ there are disjoint zero-sets $Z_{n}$ and $Z_{n}^{\prime}$ of $X$ with 


$$
f^{-1}\left[\alpha+n^{-1}, \beta-n^{-1}\right] \subset Z_{n}
$$

and

$$
f^{-1}(R \backslash(\alpha, \beta)) \subset Z_{n}^{\prime}
$$

We have

$$
f^{-1}\left[\alpha+n^{-1}, \beta-n^{-1}\right] \subset Z_{n} \subset X \backslash Z_{n}^{\prime} \subset f^{-1}(\alpha, \beta) .
$$

It follows:

$$
\bigcup_{n=1}^{\infty}\left(X \backslash Z_{n}^{\prime}\right)=f^{-1}(\alpha, \beta) .
$$

Then $f^{-1}(\alpha, \beta)$ is a countable union of cozero-sets and hence is a cozero-set.

3.4. Whenever $(X, \mathscr{Z})$ is a zero-set space, $S(X, \mathscr{L})$ will denote the set of all zero-set mappings of $X$ into $\boldsymbol{R}$, in other words, the set of zero-set functions on $X . S(X, \mathscr{L})$ will be abbreviated by $S(X)$ or $S$ where convenient. $S^{*}(X, \mathscr{L}), S^{*}(X)$ and $S^{*}$ will denote the bounded functions in $S$.

3.5. The following theorem completes the justification of our use of the terms "zero-set space" and "zero-set" with reference to the abstract structure $(X, \mathscr{z})$.

THEOREM. Let $(X, \not{2})$ be a zero-set space. Then $S(X, \mathscr{Y})$ is a uniformly closed ring of functions; it is also a lattice. The totality of zero-sets of functions in $S(X, \mathscr{Z})$ is precisely $\mathscr{z}$; i.e.,

$$
\mathscr{Z}=\{\boldsymbol{Z} f \mid f \in S(X, \mathscr{\not})\} \text {. }
$$

Corresponding statements hold for $S^{*}$.

Proof. That $S$ is a uniformly closed ring is a special case of Lemma 2.4; that $S^{*}$ is such a ring is an immediate consequence. We show $S$ and $S^{*}$ are lattices by the usual argument based on the fact that $f \in S$ implies $|f| \in S$, which follows from the definition of zero-set function in 3.1. For each $f \in S, Z f$ is the inverse image of the zero-set $\{0\}$ of $R$ and hence is in $\mathscr{Z}$. Finally we choose $Z \in \mathscr{Z}$ and find $f \in S^{*}$ with $Z f=Z$ as follows. By (5) of 2.1, there are $Z_{1}, Z_{2}, \cdots \in \mathscr{F}$ whose union is $X \backslash Z$. Recall that whenever two sets are distal in a proximity space, there is a proximity mapping into $[0,1]$ which is 0 on one of them and 1 on the other [1, Th. 25C5]. Applying this theorem to $Z$ and $Z_{n}$, we find for each $n$ a bounded proximity function $f_{n}$ with $f_{n}(Z) \subset\{0\}$ and $f_{n}\left(Z_{n}\right) \subset\{1\}$. By the pre- 
ceding lemma, $f_{n} \in S^{*}$ for all $n$. We have $Z \subset Z f_{n} \subset X \backslash Z_{n}$ and $Z=\cap\left(X \backslash Z_{n}\right)$. Hence $Z=\cap Z f_{n}$, which is the zero-set of some $f \in S^{*}$, since $\left\{Z g \mid g \in S^{*}\right\}$ is closed under countable intersection (Theorem 2.3).

3.6. If $f \in S(X)$ and $f(x) \neq 0$ for all $x \in X$, then $1 / f \in S(X)$. This statement is verified by checking that the inverse image under $1 / f$ of each open set is the inverse image under $f$ of some open set.

3.7. How to describe $S^{*}$ in terms of $S$ is clear; the following theorem describes $S$ in terms of $S^{*}$.

Theorem. Let $X$ be a zero-set space and $f: X \rightarrow \mathbf{R}$. Then the following conditions are equivalent:

(a) $f \in S$.

(b) $(f \wedge \alpha) \vee(-\alpha) \in S^{*}$ for all $\alpha>0$.

(c) There are cozero-sets $U_{1}, U_{2}, \cdots$ and $f_{1}, f_{2}, \cdots \in S^{*}$ such that:

$$
f\left|U_{n}=f_{n}\right| U_{n}
$$

for all $n$, where | denotes restriction, and

$$
\bigcup_{n=1}^{\infty} U_{n}=X
$$

Proof. $\quad(\mathrm{a}) \Rightarrow(\mathrm{b})$ is trivial since $S$ is a lattice. $(\mathrm{b}) \Rightarrow$ (c) is proved by letting $U_{n}=f^{-1}(-n, n)$ and $f_{n}=(f \wedge n) \vee(-n)$. (c) $\Rightarrow$ (a) is clear noting that for every open interval $(\beta, \gamma)$ we have:

$$
f^{-1}(\beta, \gamma)=\bigcup_{n=1}^{\infty}\left[U_{n} \cap f_{n}^{-1}(\beta, \gamma)\right]
$$

which clearly is a cozero-set.

3.8. Before proceeding to the next topic, we digress for a moment to discuss the relationship between zero-set structures and proximity structures. As we have seen, each zero-set structure gives rise to a proximity structure. If we take the totality of zero-sets of the proximity functions for this latter structure, we recover the former one. However, not every proximity structure arises from a zero-set structure. For example, the topological space $\boldsymbol{R}$ of real numbers admits only one zero-set structure. In more general terms, a proximity structure arising from a zero-set structure has the property that its proximity functions form a ring, but an arbitrary proximity structure need not have this property. In fact, we have here essentially the same situation which was discussed in the introduction. It can be shown easily that the proximity structures on a set $X$ are in a natural one-to-one correspondence with those uniformly closed 
rings of bounded functions on $X$ which contain the constants and separate points. The zero-sets of the functions in any such ring give us a zero-set structure, but the same zero-set structure can arise from more than one ring. If, starting with a zero-set sturcture, we take the largest ring having the given zero-sets, the proximity structure corresponding to this largest ring is the one defined above.

\section{Zero-set and uniform structures.}

4.1. Let $X$ be a zero-set space. For each $f \in S(X)$, we define as usual a pseudometric on $X$ by

$$
p_{f}(x, y)=|f(x)-f(y)| \text {. }
$$

The uniform structure defined by $\left\{p_{f} \mid f \in S\right\}$ will be denoted by $U(X)$ or simply $U$, while that defined by $\left\{p_{f} \mid f \in S^{*}\right\}$ will be denoted by $U^{*}(X)$ or $U^{*}$.

THEOREM 4.2. The proximity structure on $X$ defined in 3.2. is the one induced on $X$ by $U^{*}$ and also the one induced by $U$.

Proof. Recall that that $A$ and $B$ are distal for the proximity structure determined by a uniform structure means that for some symmetric entourage $V$ no element of $A$ is a neighbor of order $V$ of any element of $B$, in symbols $V(A) \cap B=\phi$.

Suppose $A$ and $B$ are distal for the structure defined in 3.2. As before, [1, Th. 25C5] implies there is a bounded proximity function $f$ such that $f(A) \subset\{0\}$ and $f(B) \subset\{1\}$. We know $f \in S^{*}$ (Lemma 3.3). Thus

$$
\boldsymbol{V}=\left\{(x, y) \mid p_{f}(x, y)<1\right\},
$$

which is an entourage for both $U$ and $U^{*}$, serves to show $A$ and $B$ are distal for both the proximity structure determined by $U$ and that determined by $U^{*}$.

Conversely suppose $A$ and $B$ are distal for either the proximity structure determined by $U$ or that determined by $U^{*}$. Then for some entourage $\boldsymbol{V}$ of $U$ or $U^{*}, x \in A$ and $y \in B$ imply $(x, y) \notin V$. In either case, there are $f_{1}, \cdots, f_{n} \in S$ and $\varepsilon_{1}, \cdots, \varepsilon_{n}>0$ such that:

$$
\boldsymbol{V} \supset\left\{(x, y)|| f_{i}(x)-f_{i}(y) \mid \leqq \varepsilon_{i} \text { for all } i\right\} .
$$

Let $g_{i}=\left(1 / \varepsilon_{i}\right) f_{i}$ for each $i$. Then whenever $x \in A$ and $y \in B$,

$$
\left|g_{i}(x)-g_{i}(y)\right| \geqq 1
$$

for some $i$. Consider $g: X \rightarrow \boldsymbol{R}^{n}$ defined by 


$$
g(x)=\left(g_{1}(x), \cdots, g_{n}(x)\right) .
$$

Note $d(g A, g B) \geqq 1$, where $d$ is of course the distance function on $\boldsymbol{R}^{n}$. Define $\varphi: \boldsymbol{R}^{n} \rightarrow \boldsymbol{R}$ by

$$
\varphi(t)=\min \{d(t, g B), 1\}
$$

and note $\varphi$ is continuous. We show $\varphi \circ g$ is a zero-set function on $X$. If $U$ is open in $R, \varphi^{-1} U$ is open in $R^{n}$, and hence is a countable union of sets of the form $V_{1} \times \cdots \times V_{n}$ with each $V_{i}$ open. For each such set,

$$
g^{-1}\left(V_{1} \times \cdots \times V_{n}\right)=g_{1}^{-1} V_{1} \cap \cdots \cap g_{n}^{-1} V_{n}
$$

is a zero-set of $X$, hence $\varphi \circ g$ is indeed a zero-set function. But $(\varphi \circ g)(x)=0$ if $x \in B$ while $(\varphi \circ g)(x)=1$ if $x \in A$. Thus $A$ and $B$ are contained in the disjoint zero-sets $(\varphi \circ g)^{-1}(0)$ and $(\varphi \circ g)^{-1}(1)$ respectively. Thus $A$ and $B$ are distal for the uniform structure defined in 3.2.

4.3. In connection with the following theorem, we note that it will be apparent later that every function on $X$ uniformly continuous for $U^{*}$ is bounded. We emphasize that we are using the standard structures of $\boldsymbol{R}$ in the theorem.

THEOREm. Let $f$ be a real-valued function on $X$. The following statements are equivalent:

(a) $f$ is a zero-set mapping.

(b) $f$ is a proximity mapping.

(c) $f$ is uniformly continuous for $U$.

For bounded $f$, each of the preceding conditions is equivalent to:

(c*) $f$ is uniformly continuous for $U^{*}$.

Proof. Both $(\mathrm{c}) \Rightarrow(\mathrm{b})$ and $\left(\mathrm{c}^{*}\right) \Rightarrow(\mathrm{b})$ since a uniformly continuous mapping is always a proximity mapping for the proximity structures determined by the uniform structures on the two spaces involved. (b) $\Rightarrow$ (a) is part of Lemma 3.3. (a) $\Rightarrow$ (c) is obvious from the definition of $U$. Similarly, if $f$ is bounded and satisfies (a), it satisfies $\left(\mathrm{c}^{*}\right)$ by the definition of $U^{*}$.

4.4. At the risk of undue repetition, we point out that the preceding theorem is not a special case of the following one. While it is true that we may take $Y=\boldsymbol{R}$ as a zero-set space, neither $U(\boldsymbol{R})$ nor $U^{*}(\boldsymbol{R})$ is the standard uniform structure of $\boldsymbol{R}$.

THeOREm. Let $X$ and $Y$ be zero-set spaces and $\varphi: X \rightarrow Y$. Then the following statements are equivalent: 
(a) $\varphi$ is a zero-set mapping.

(b) $\varphi$ is a proximity mapping.

(c) $\phi$ is uniformly continuous for $U(X)$ and $U(Y)$.

$\left(\mathrm{c}^{*}\right) \varphi$ is uniformly continuous for $U^{*}(X)$ and $U^{*}(Y)$.

Proof. $(\mathrm{c}) \Rightarrow(\mathrm{b})$ and $\left(\mathrm{c}^{*}\right) \Longrightarrow(\mathrm{b})$ are a general property of uniform spaces as in the preceding theorem. $(a) \Rightarrow\left(c^{*}\right)$ is proved as follows: $U^{*}(Y)$ is defined by pseudometrics of the form $p_{g}$ with $g \in S^{*}(Y)$. Since $\varphi$ is a zero-set mapping, $g \circ \varphi \in S^{*}(X)$. Thus

$$
\left(x_{1}, x_{2}\right) \rightarrow p_{g}\left(\varphi\left(x_{1}\right), \varphi\left(x_{2}\right)\right)=p_{g \circ \varphi}\left(x_{1}, x_{2}\right)
$$

is one of the pseudometrics which defines $U^{*}(X) . \quad(\mathrm{a}) \Rightarrow(\mathrm{c})$ is proved similarly. Finally we prove $(b) \Rightarrow(a)$ : Let $Z$ be a zero-set of $Y$. Then $Z=Z g$ for some $g \in S(Y)$. The preceding theorem implies first that $g$ is a proximity mapping and then, since it follows $g \circ \varphi$ is also one, that $g \circ \varphi \in S(X)$. Thus $\varphi^{-1} Z=Z(g \circ \varphi)$ is a zero-set of $X$.

\section{Compactification of zero-set spaces.}

5.1. Some definitions are necessary before we can begin constructing compactifications. Let $X$ be a zero-set space and $A \subset X$. Consider the collection $\mathscr{Z}_{A}$ of all sets of the form $Z \cap A$ where $Z$ is a zero-set of $X$. Clearly this collection is closed under countable intersection and finite union. Let $\mathscr{C}$ be the set of restrictions to $A$ of zero-set functions on $X$. Then every set in $\mathscr{Z}_{A}$ is the zero-set of a function in $\mathscr{C}$. On the other hand, the inverse image of each closed set of $\boldsymbol{R}$ under a function in $\mathscr{C}$ is in $\mathscr{Z}_{A}$. Thus by Lemma 2.5, $\mathscr{F}_{A}$ is a zero-set structure on $A$. We call this structure the relative zero-set structure induced on $A$ by $X$ and refer to $A$ with this structure as a zero-set subspace of $X$.

5.2. We call a zero-set space $(X, \mathscr{z})$ compact if the associated topological space is compact. Note that this definition is equivalent to defining $X$ to be compact if every covering of $X$ by cozero-sets has a finite sub-covering. Given a compact zero-set space $X, S(X)$ is a uniformly closed ring of continuous functions which separates points and contains the constants. Thus $S$ is exactly the set of all continuous functions on $X$ by the Stone-Weierstrass Theorem. In short, $S$ is determined by the topology. Since $\mathscr{Z}$ consists of all zero-sets of functions in $S$, we conclude that a compact topological space admits a unique zero-set structure.

5.3. By a compactification of a zero-set space $X$ we mean, of course, a compact space $Y$-whether we describe $Y$ as a zero-set 
space or a topological space is immaterial in view of the preceding paragraph-which has $X$ as a dense zero-set subspace. After a few more definitions, we will explain how it is known that every zero-set space has a compactification.

5.4. Let $(X, \mathscr{Z})$ be a zero-set space. We call $\mathscr{A}$ a $z$-filter on $X$ if $\mathscr{A}$ is a nonempty subset of $\mathscr{Z}$ such that:

(1) $\varnothing \notin \mathscr{A}$.

(2) If $A \in \mathscr{A}, B \in \mathscr{Z}$ and $B \supset A$, then $B \in \mathscr{A}$.

(3) If $A$ and $B \in \mathscr{A}$, then $A \cap B \in \mathscr{A}$.

A $z$-filter which is not contained in any other $z$-filter is called a $z$-ultrafilter. A $z$-ultrafilter is called real if it is closed under countable intersection. A $z$-ultrafilter is called hyperreal if it contains a countable collection of sets whose intersection is empty. Certainly, no $z$-ultrafilter can be both real and hyperreal; we check that each one is either real or hyperreal. Let $\mathscr{A}$ be a $z$-ultrafilter which is not hyperreal. Let $\mathscr{A}^{\prime}$ be the totality of countable intersections of sets chosen from $\mathscr{A}$. Then it is easy to verify that $\mathscr{A}^{\prime}$ is a $z$-filter and $\mathscr{A}^{\prime} \supset \mathscr{A}$. Hence $\mathscr{A}=\mathscr{A}^{\prime}$ and $\mathscr{A}$ is real.

5.5. Let us return for a moment to a situation described earlier. Let $X$ be a set and $\mathscr{R}$ be a ring of bounded functions on $X$. We suppose $\mathscr{R}$ is uniformly closed, contains the constants and separates points. There are several well-known ways to construct a compact Hausdorff topological space $\hat{X}$ having the following properties:

(1) The set $X$ is dense in $\hat{X}$.

(2) Each function in $\mathscr{R}$ has a continuous extension to $\hat{X}$.

(3) The restriction to $X$ of each continuous function on $\hat{X}$ is in $\mathscr{R}$.

These properties serve to uniquely determine $\hat{X}$. Among the methods of constructing $\hat{X}$, the ones most relevant to the discussion below are those of Lorch $[5, \S 3]$ and of the author [3, §2].

5.6. Continuing with $X$ and $\mathscr{R}$, we make $X$ into a zero-set space by defining the zero-sets to be the zero-sets of the functions in $\mathscr{R}$ (Theorem 2.3). (The special case where $S^{*}(X)=\mathscr{R}$ will be discussed in the next section.) The same zero-set structure on $X$ is obtained by putting on $\hat{X}$ the unique zero-set structure admitted by its topology and regarding $X$ as a subspace of $\hat{X}$. Thus $\hat{X}$ is a compactification of $X$ as a zero-set space. Furthermore every compactification $Y$ of a zero-set space $X$ may be obtained in this way by letting $\mathscr{R}$ be the set of all restrictions to $X$ of continuous functions on $Y$. 
5.7. Starting with $X$ and $\mathscr{R}$ again, we define $\hat{X}$ and recall [3, Th. 3.2]:

THEOREM. Each z-ultrafilter on $X$ converges, as a filter base on $\hat{X}$, to some $p \in \hat{X}$. Each $p \in \hat{X}$ is the limit of some z-ultrafilter.

(We warn the reader that in the general situation under consideration here it is possible for $p \in \hat{X}$ to be the limit of more than one $z$-ultrafilter.)

5.8. Lorch [5, §3] has distinguished two classes of points of $\hat{X}$. These classes reflect the notion of real and hyperreal $z$-ultrafilter as introduced in 5.4. Specifically, we have the following theorem, taken from [3, Ths. 4.7 and 4.8].

THEOREM. Let $p \in \hat{X}$ and $\mathscr{A}$ be a z-ultrafilter converging to $p$. If there exists an $f \in \mathscr{R}$ such that the continuous extension of $f$ to $\hat{X}$ vanishes at $p$ but $f(x) \neq 0$ for all $x \in X$, then $\mathscr{A}$ is hyperreal. If no such $f$ exists, then $\mathscr{A}$ is real.

\section{6. $\beta X$ and $v X$ for a zero-set space $X$.}

6.1. A special case of the discussion of the preceding section arises when we start with a zero-set space $X$ and set $\mathscr{R}=S^{*}(X)$. In this case we denote $\hat{X}$ by $\beta X$. As the notation suggests, $\beta X$ is a natural generalization of the Stone-Čech compactification of a completely regular space. In particular, as we have seen, $\beta X$ is a compactification of $X$ as a zero-set space and every zero-set function on $X$ has a continuous (i.e., by 5.2, zero-set) extension to $\beta X$. Additional properties of the Stone-Čech compactification which apply to our $\beta X$ will become apparent as we proceed. We note at once that every point of $\beta X$ is the limit of a unique $z$-ultrafilter on $X$ by [3, Th. 3.3].

6.2. We denote by $\cup X$ the zero-set subspace of $\beta X$ consisting of those points which are limits of real $z$-ultrafilters on $X$. Towards verifying that $X \subset v X$, let $\mathscr{A}^{x}$ be the $z$-ultrafilter converging to some $x \in X$. We show that $\mathscr{A}^{x}$ consists of those zero-sets of $X$ which contain $x$ and hence is real. Since these zero-sets form a $z$-filter, it suffices to show $\mathscr{A}^{x}$ contains no other sets. But this is evident since $f\left(\mathscr{A}^{x}\right)$ converges to $f(x)$ for every $f \in S^{*}(X)$ and hence $\boldsymbol{Z} f \in \mathscr{A}^{x}$ implies $f(x)=0$, i.e., $x \in \boldsymbol{Z} f$. Thus it is clear that $X$ is a dense zero-set subspace of $v X$. That $v X$ is a natural generalization of the Hewitt real compactification of a completely regular space will be developed in detail below. 
THEOREM 6.3. Every $f \in S(X)$ has an extension in $S(\nu X)$. However if $p \in \beta X \backslash \cup X$, there is an $f \in S(X)$ which has no extension to $X \cup\{p\}$ which is even continuous.

Proof. Let $f \in S(X)$. Let $g_{1}=1 /\left(f^{+}+1\right), g_{2}=1 /\left(f^{-}+1\right)$. Then $g_{1}, g_{2} \in S^{*}(X)$ by 3.5 and 3.6. Thus $g_{1}, g_{2}$ have zero-set extensions to $\beta X$; let $h_{1}, h_{2}$ be the restrictions to $\cup X$ of these extensions. By Theorem 5.8, since $g_{1}$ and $g_{2}$ are never zero on $X$, they are never zero on $v X$. Thus $1 / h_{1}-1 / h_{2}$ is defined; clearly it is the required extension of $f$.

Now suppose $p \in \beta X \mid \cup X$. Then there is a $g \in S^{*}(X)$ such that the continuous extension of $g$ to $\beta X$ vanishes at $p$ but $g(x) \neq 0$ for all $x \in X$. Let $f=1 / g$ and note $f \in S(X)$. But, the $z$-ultrafilter $\mathscr{A}^{p}$ which converges to $p$ is such that $g\left(\mathscr{A}^{p}\right)$ converges to 0 and hence $f\left(\mathscr{C}^{p}\right)$ cannot converge. Thus $f$ has no continuous extension to $X \cup\{p\}$.

6.4. We now show that $\beta X$ and $\cup X$ are the completions of $X$ for $U^{*}(X)$ and $U(X)$ respectively. Of course, the compact space $\beta X$ is the completion of $X$ for some uniform structure; we omit the routine checking of definitions which verifies that this structure is indeed $U^{*}(X)$.

Theorem. $(v X, U(u X))$ is the completion of $(X, U(X))$.

Proof. Again it is necessary to check definitions in a manner too tedious to record here. We need to know that $U(v X)$ and $U^{*}(\beta X)$ induce on $X$ the relative uniform structures $U(X)$ and $U^{*}(X)$ respectively. We also use the identity of the relative topology induced on $v X$ by $\beta X$ with the topology defined by $U(v X)$. We conclude then that $X$ is dense in $v X$. Thus we need only show that every Cauchy filter for $U(X)$ has a limit in $v X$. Let $\mathscr{F}$ be such a Cauchy filter. Then, since $U^{*}(X)$ is coarser than $U(X), \mathscr{F}$ has a limit $p$ in the compact space $\beta X$. Suppose $p \notin v X$. Then there is a continuous function $f$ on $\beta X$ such that $f(p)=0$ but $f(x) \neq 0$ for all $x \in X$. Let $g$ be the reciprocal of the restriction of $f$ to $X$ and note $g \in S(X)$. Since $g$ is uniformly continuous on $(X, U(X))$ by the definition of $U(X), g(\mathscr{F})$ is a Cauchy filter base on $\boldsymbol{R}$. But since $f(\mathscr{F})$ converges to $f(p)=0, g(\mathscr{F})$ can not converge. This contradiction establishes $p \in \cup X$ and completes the proof.

COROLlaRy 6.5. Let $X$ and $Y$ be zero-set spaces and $\varphi: X \rightarrow Y$ be a zero-set mapping. Then $\varphi$ has extensions $\varphi^{\circ}$ and $\varphi^{\beta}$ which are zero-set mappings with $\phi^{v}: \cup X \rightarrow \cup Y$ and $\varphi^{\beta}: \beta X \rightarrow \beta Y$. 
Proof. By Theorem 4.4, $\varphi$ is uniformly continuous for $U(X)$ and $U(Y)$; hence it has a uniformly continuous extension $\varphi^{\circ}$ mapping the completion of $(X, U(X))$ into that of $(Y, U(Y))$. But by the preceding theorem and 4.4 again, $\varphi^{\prime \prime}$ is as required. The part about $\phi^{\beta}$ is similar.

\section{Pseudocompact and realcompact spaces.}

7.1. Pseudocompactness and realcompactness are defined in the obvious way. Explicitly, a zero-set space $X$ is called realcompact if every real $z$-ultrafilter on $X$ converges to a point of $X$, i.e., if $v X=X$. $X$ is called pseudocompact if it has no hyperreal $z$-ultrafilter, i.e., if $v X=\beta X$.

7.2. In other theories, many conditions are known to be equivalent to pseudocompactness. For reasons which will become apparent as we proceed, these conditions carry over essentially unchanged to the present context. We merely refer the reader to Gillman and Jerison [2] and Lorch [5]. We do record here two corollaries for future use. Both are due to Lorch [5; Ths. 11 and 9], who states them in quite different language; both are special cases of Theorem 5.8 above.

COROLlaRY 7.3. Let $X$ be a zero-set space and $Y$ be any compactification of $X$. Then $X$ is pseudocompact if and only if every nonempty zero-set of $Y$ meets $X$. In other words, if and only if each continuous function on $Y$ which vanishes nowhere on $X$ vanishes nowhere on $Y$.

CoRollary 7.4. Let $X$ be a zero-set space and $Y$ be any compactification of $X$. Then $X$ is realcompact if and only if each $p \in Y \backslash X$ is contained in a zero-set of $Y$ which does not meet $X$. In other words, if and only if for each $p \in Y$ there is a continuous $f$ on $Y$ with $f(p)=0$ but $f(x) \neq 0$ for all $x \in X$.

7.5. We place here, for the record, a few significant facts, which are trivial consequences of what we have already proved. That $\beta(\beta X)=\beta X$ and $\nu(\beta X)=\beta X$ requires no explanation. $\beta(\nu X)=\beta X-$ equal in the sense of isomorphic-since both are compactifications of $X$ having essentially the same ring of continuous functions. Next we apply Corollary 7.4 to $\beta(v X)=\beta X$ as a compactification of $v X$. Noting that $f(x) \neq 0$ for all $x \in X$ implies $f(q) \neq 0$ for all $q \in v X$, we see that $v X$ is realcompact. In other words $v(v X)=v X$. 
It is well-known that a topological space $X$ is compact if and only if every multiplication linear functional on $C^{*}(X)$ is of the form $f \rightarrow f(x)$ for some $x \in X$; here, as throughout the paper, we exclude the zero functional from the multiplicative linear functionals. As a result, the same statement holds for a zero-set space $X$ and $S^{*}(X)$. We prove the corresponding statement for realcompactness and $S(X)$ next. Clearly if $X$ is not realcompact, we need merely choose $p \in u X \backslash X$ and consider $f \rightarrow f^{\prime}(p)$, where $f^{\nu}$ denotes the continuous extension of $f$ to $v X$, to find a multiplicative linear functional on $S(X)$ which does not arise from a point of $X$. Suppose conversely $X$ is realcompact. Let $P: S(X) \rightarrow \boldsymbol{R}$ be a multiplicative linear functional. The restriction of $P$ to $S^{*}(X)$ is such a functional on $S^{*}(X)$, and hence there is a $p \in \beta X$ with $P f=f^{3}(p)$ for all $f \in S^{*}(X)$, where $f^{\beta}$ denotes the continuous extension of $f$ to $\beta X$. If $p$ were in $\beta X \backslash \cup X=$ $\beta X \backslash X$, there would be an $f \in S^{*}(X)$ such that $P f=f^{\beta}(p)=0$ which vanished nowhere on $X$, and thus, by 3.6, with $1 / f \in S(X)$. It follows $p \in X$. In short, $X$ is realcompact if and only if every multiplicative linear functional on $S(X)$ is of the form $f \rightarrow f(x)$ for some $x \in X$.

THEOREM 7.6. A zero-set space $X$ is pseudocompact if and only if each zero-set function on $X$ is bounded.

Proof. Since, as noted above (3.6), the reciprocal of a zero-set function is also one, the existence of an unbounded zero-set function is equivalent to the existence of a bounded zero-set function $f$ with $f(x)>0$ for all $x \in X$ but inf $\{f(x) \mid x \in X\}=0$. Such functions $f$ may be described as zero-set functions whose continuous extensions to $\beta X$ vanish somewhere on $\beta X$ but nowhere on $X$. By Corollary 7.3, the result is now clear.

7.7. The following theorem is to be contrasted with the topological space case, where the union of two realcompact subspaces may fail to be realcompact [2, Exercise $8 \mathrm{H} 6$ ].

THEOREm. Let $A_{1}, A_{2}, \cdots \subset X$ all be realcompact. Then $A=\cup A_{n}$ is realcompact.

Proof. Consider the compactification $B=\operatorname{cl}_{\beta X} A$ of $A$, and let $p \in B \backslash A$. For each $n$ with $p \notin \mathrm{cl}_{\beta X} A_{n}$, we can use complete regularity to find a continuous $f_{n}: \beta X \rightarrow \boldsymbol{R}$ such that $f_{n}(p)=0$ but $f_{n}(x) \neq 0$ for all $x \in A_{n}$. But for each $n$ with $p \in \operatorname{cl}_{\beta X} A_{n}$, we can use Corollary 7.4 to find a continuous $g_{n}: \operatorname{cl}_{\beta X} A_{n} \rightarrow \boldsymbol{R}$ with $g_{n}(p)=0$ but $g_{n}(x) \neq 0$ for each $x \in A_{n}$; then extending $g_{n}$ from the compact space $\operatorname{cl}_{\beta X} A_{n}$ to all 
of $\beta X$, we again find a continuous $f_{n}: \beta X \rightarrow \boldsymbol{R}$ such that $f_{n}(p)=0$ but $f_{n}(x) \neq 0$ for all $x \in A_{n}$. Let:

$$
f=\sum_{n=1}^{\infty} 2^{-n}\left(\left|f_{n}\right| \wedge 1\right) .
$$

Then $f(x) \neq 0$ for all $x \in A$, but $f(p)=0$. Restricting $f$ to $\operatorname{cl}_{\beta X} A$ and using Corollary 7.4, we have the desired result.

7.8. The following theorem also holds for topological spaces [2, Th. 8.9]:

TheOREM. Let $\left(A_{\imath}\right)_{\iota \in I}$ be a family of realcompact subspaces of $X$. Then $\cap A_{\text {c }}$ is realcompact.

Proof. Consider the compactification $B=\operatorname{cl}_{\beta X} \cap A_{\iota}$ of $\cap A_{\iota}$, and let $p \in B \backslash \cap A_{\iota}$. Then for some $\iota, p \notin A_{\iota}$. But $p \in \operatorname{cl}_{\beta X} A_{\iota}$ and hence there is a continuous $f: \operatorname{cl}_{\beta X} A_{\iota} \rightarrow \boldsymbol{R}$ with $f(p)=0$ but $f(x) \neq 0$ for all $x \in A_{\iota}$. Restricting $f$ to $B$, we conclude $\cap A_{\iota}$ is realcompact.

THEOREM 7.9. Let $Y$ be a realcompact zero-set space and $X \subset Y$. Then there is an $X^{\prime} \subset Y$ which is $\cup X$ in the sense that it is isomorphic as a zero-set space to $\cup X$ under an isomorphism leaving $X$ pointwise fixed.

Proof. Let $i: X \rightarrow Y$ be the inclusion mapping. By Corollary 6.5, $i$ has a zero-set extension $i^{\prime}: v X \rightarrow Y$. we show that $i^{\nu}$ is injective. Let $p_{1}$ and $p_{2}$ be distinct points of $v X$. Then $p_{1}$ and $p_{2}$ are the respective limits of distinct real $z$-ultrafilters $\mathscr{A}_{1}$ and $\mathscr{A}_{2}$ on $X$. Since $i \mathscr{A}_{j}=\mathscr{A}_{j}, \mathscr{A}_{j}$ converges to $i^{v} p_{j} \in Y(j=1,2)$. But by [3, Th. 4.7], the distinct real $z$-ultrafilters $\mathscr{A}_{1}$ and $\mathscr{A}_{2}$ have distinct limits in the compactification $\operatorname{cl}_{\beta X} X$ of $X$. In short, $i^{\nu} p_{1} \neq i^{\nu} p_{2}$ and $i^{\nu}$ is indeed injective.

It remains only to set $X^{\prime}=i^{\nu}(\nu X)$ and to show that $i^{\nu} Z$ is a zero-set of $X^{\prime}$ for an arbitrary zero-set $Z$ of $\cup X$. Since $Z \cap X$ is a zero-set of $X$, a subspace of $X^{\prime}, Z \cap X=Z^{\prime} \cap X$ for some zero-set $Z^{\prime}$ of $X^{\prime}$. Choosing $f \in S\left(X^{\prime}\right)$ such that $Z f=Z$, we note that

$$
i^{v} \boldsymbol{Z}\left(f \circ i^{\nu}\right)=Z f=Z^{\prime}
$$

is a zero-set of $X^{\prime}$; thus we need only show that $Z\left(f \circ i^{\prime}\right)=Z$. To this end, it suffices to show that whenever $Z_{1}$ and $Z_{2}$ are zero-sets of $v X$ having the same intersection with $X$, then $Z_{1}=Z_{2}$. Suppose on the contrary we have such $Z_{1}$ and $Z_{2}$ and $p \in Z_{1}$ but $p \notin Z_{2}$. Then there is a $g \in S^{*}(\nu X)$ with $g(p)=1, Z g=Z_{2}$ and $g \leqq 1$. Also there is an $h \in S^{*}(\nu X)$ with $h \geqq 0$ and $Z h=Z_{1}$. Then $1-g+h$ can vanish 
only at those $q$ where $h(q)=0$ and $g(q)=1$, and hence $q \in Z_{1}$ while $q \notin Z_{2}$. Thus $1-g+h$ never vanishes on $X$. But it does vanish at $p$, which is impossible by Theorem 5.8 applied to the compactification $\beta(\cup X)=\beta X$ of $X$. This contradiction completes the proof.

CoROllary 7.10. A closed subspace of a realcompact zero-set space is realcompact.

Corollary 7.11. Let $X$ be a subspace of a zero-set space $Y$. Suppose each real z-ultrafilter of $X$ has a limit in $Y$ and each point of $Y$ is the limit of such a z-ultrafilter. Then $Y$ is (isomorphic to) $\cup X$.

Proof. We have $X \subset Y \subset \cup Y$, and thus, identifying the $X^{\prime}$ of the theorem with $v X, X \subset v X \subset v Y$. By definition of $v X$, the points of $v X$ are exactly the limits of the real $z$-ultrafilters of $X$. But by hypothesis then, $v X=Y$.

CoRollary 7.12. Let $X$ be a subspace of a realcompact zero-set space $Y$. If each point of $Y$ is the limit of a real $z$-ultrafilter of $X$, then $Y$ is (isomorphic to) $\cup X$.

Proof. We repeat the preceding proof noting that $Y=v Y$.

THEOREM 7.13. Let $X$ be a subspace of a realcompact zero-set space $Y$. In order that $Y$ be (isomorphic to) $\cup X$ it is necessary and sufficient that each nonempty zero-set of $Y$ meet $X$.

Proof. Necessity. We must show that each nonempty zero-set $Z$ of $v X$ meets $X$. Choosing $f \in S^{*}(v X)$ with $Z f=Z$ and applying Theorem 5.8 to the compactification $\beta(\nu X)=\beta X$, we have the desired conclusion.

Sufficiency. We have $X \subset Y \subset \beta Y$ as zero-set spaces. We show first that $X$ is dense in $\beta Y$. If not, we may use the complete regularity of $\beta Y$ to find a continuous, hence zero-set, function $f$ on $\beta Y$ with $X \subset Z_{f}$ but $f(p)=1$ for some $p \in \beta Y$. Let

$$
Z^{\prime}=\{q \in \beta X \mid f(q) \geqq 1 / 2\}
$$

and note, since $Y$ is dense in $\beta Y, Z=Z^{\prime} \cap Y$ is nonempty. But then the nonempty zero-set $Z$ of $Y$ does not meet $X$, contrary to hypothesis. Thus $X$ is indeed dense in $\beta Y$.

It follows by Theorem 5.7 that each point of $Y$, in fact each point of $\beta Y$, is the limit of some $z$-ultrafilter of $X$. Suppose some 
$p \in Y$ is the limit of a hyperreal $z$-ultrafilter of $X$. Then by Theorem 5.8 there is a zero-set $Z$ of $\beta Y$ which contains $p$ but does not meet $X$. But then $Z \cap Y$ is a nonempty zero-set of $Y$ which does not meet $X$, contrary to hypothesis. Thus every $p \in Y$ is the limit of a real $z$-ultrafilter of $X$. The theorem now follows from the preceding corollary.

7.14. The "if" part of the following theorem holds also for topological spaces, but the "only if" part fails.

THEOREM. A zero-set space $X$ is pseudocompact if and only if it has only one compactification. (In other words, if and only if any two compactifications are homeomorphic under a homeomorphism lsaving $X$ pointwise fixed.)

Proof. If: Suppose $X$ is not pseudocompact. By essentially the same argument used in [2, Exercise 9D2], $\beta X$ contains at least $2^{C}$ (where $C=2^{\boldsymbol{N}_{0}}$ ) points which are each the limit of a hyperreal $z$ ultrafilter. Let $p_{1}$ and $p_{2}$ be two such points. Let $\mathscr{R}$ consist of those functions $f \in S^{*}(X)$ whose continuous extension $f^{\beta}$ to $\beta Y$ have the property that $f^{3}\left(p_{1}\right)=f^{\beta}\left(p_{2}\right)$. Now construct $\hat{X}$ for $X$ and $\mathscr{R}$ as described in 5.5. Since the restriction to $X$ of each continuous $h$ on $\beta X$ with $h\left(p_{1}\right) \neq h\left(p_{2}\right)$ admits no continuous extension to $\hat{X}, \hat{X}$ and $\beta X$ are certainly distinct (in the sense specified in the statement of the theorem). It only remains to show that $\hat{X}$ is a compactification of $X$ as a zero-set space; i.e., since $\hat{X}$ is compact and $X$ is dense in $\hat{X}$, that $X$ with its initial zero-set structure is a zero-set subspace of $\hat{X}$. In other words, that

$$
\{\boldsymbol{Z} f \mid f \in \mathscr{R}\}=\left\{\boldsymbol{Z} f \mid f \in S^{*}(X)\right\} .
$$

Since $\mathscr{R} \subset S^{*}(X)$, we complete the proof by showing that for each $g \in S^{*}(X)$ there is an $f \in \mathscr{R}$ with $\boldsymbol{Z} f=\boldsymbol{Z} g$. But since $p_{1}$ and $p_{2}$ are limits of hyperreal $z$-ultrafilters, there are $g_{1}$ and $g_{2} \in S^{*}(X)$ whose continuous extensions to $\beta X$ vanish at $p_{1}$ and $p_{2}$ respectively, but which never vanish on $X$. Then clearly $g_{1} g_{2} g \in \mathscr{R}$, while $\boldsymbol{Z}\left(g_{1} g_{2} g\right)=\boldsymbol{Z} g$; which completes the proof of "if".

Only if: Suppose $X$ is pseudocompact and let $Y$ be any compactification of $X$. By Theorem 7.9, there is an $X^{\prime}$ isomorphic to $v X$ with $X \subset X^{\prime} \subset Y$. Since $v X=\beta X, X^{\prime}$ is compact and hence closed in $Y$. Hence $X^{\prime}=Y$; in other words, $v X$ is the only compactification of $X$.

7.15. In order to prove theorems about products, we must first define the product of a family $\left(X_{t}\right)_{\iota \in I}$ of zero-set spaces. The zero-sets 
of $X=\Pi X_{\iota}$ will be the obvious ones, namely all sets which are countable intersections of finite unions of sets of the form $\operatorname{pr}_{\iota}^{-1} Z_{\iota}$ where $Z_{\iota}$ is a zero-set of some $X_{\iota}$ and $\mathrm{pr}_{\iota}$ is the projection of $X$ on $X_{\iota}$. But we must verify that these sets indeed form a zero-set structure on $X$. To this end, let $\mathscr{z}_{0}$ be the collection of all sets of the form $\operatorname{pr}_{\iota}^{-1} Z_{\iota}$ as above; let $\mathscr{Z}$ be the collection of all countable intersections of finite unions of such sets. By Lemma 2.5, it suffices to show that for each $Z_{0} \in \mathscr{Z}_{0}$, there is a function $f$ on $X$ such that $Z f=Z_{0}$ and $f^{-1} F \in \mathscr{Z}$ for each $F$ closed in $R$. But if $Z_{0} \in \mathscr{Z}_{0}, Z_{0}=\mathrm{pr}_{\iota}^{-1} Z$ where $Z=Z g$ for some zero-set function $g$ on $X_{c}$. It suffices then to let $f=g \circ \mathrm{pr}$, to complete the proof that $\mathscr{Z}$ is a zero-set structure on $X$. It is obvious that the topology induced on $X$ by the zero-set structure $\approx 2$ is the product of the topologies induced on the $X_{\iota}$ by their zero-set structures.

7.16. The following theorem holds also for topological spaces [2, Th. 8.11].

THEOREM. Let $\left(X_{t}\right)_{\iota \in I}$ be a family of realcompact zero-set spaces. Then $X=\Pi X_{\iota}$ is realcompact.

Proof. We use Corollary 7.4 applied to $Y=\Pi \beta X_{\iota}$ as a compactification of $X$. Let $p \in Y \backslash X$. Then for some $\iota, p_{\iota}=\operatorname{pr}_{\iota} p \notin X_{\iota}$. By the corollary applied to $X_{\iota}$ and $\beta X_{\iota}$, there is a continuous $f$ on $\beta X_{\iota}$ which vanishes nowhere on $X_{\iota}$ such that $f\left(p_{\iota}\right)=0$. Since $f \circ \mathrm{pr}_{\iota}$ vanishes nowhere on $X$ but does vanish at $p$, the theorem is proved.

7.17. The following theorem and corollary are to be contrasted with [2, Exercise 9I and 9.15], where counterexamples to the corresponding statements for topological spaces are to be found; these counterexamples involve products of just two factors.

THEOREM. Let $\left(X_{\epsilon}\right)_{\epsilon \in I}$ be a family of zero-set spaces, let $X=\Pi X_{\iota}$ and let $Y=\Pi \cup X_{\iota}$. Then $Y=\cup X$.

Proof. By the preceding theorem, $Y$ is realcompact. By Theorem 7.13 then, we need only show that every nonempty zero-set of $Y$ meets $X$. Let $Z$ be such a zero-set; $Z$ is a countable intersection of finite unions of sets of the form $\operatorname{pr}_{t}^{-1} Z_{t}$ with $Z_{t}$ a zero-set of $v X_{t}$. Fixing $p \in Z$, we replace each of these finite unions by one of the sets whose union is taken, choosing this one to contain $p$. In this way, we find $Z^{\prime} \subset Z$ such that $Z^{\prime}$ is nonempty and is a countable intersection of sets of the form $\operatorname{pr}_{c}^{-1} Z_{\iota}$. Thus we may write: 


$$
Z^{\prime}=\bigcap_{n=1}^{\infty} \operatorname{pr}_{\iota_{n}}^{-1} Z_{\iota_{n}}
$$

where $Z_{\iota_{n}}$ is a zero-set of $U X_{\iota_{n}}$ and, without loss of generality, suppose that $\iota_{1}, \iota_{2}, \ldots$ are distinct. Since each nonempty zero-set of $v X_{c}$ meets $X_{\iota}$ (Theorem 7.3), we may choose, for each $n$, an $x_{n} \in Z_{\iota_{n}} \cap X_{\iota_{n}}$. Choosing any $x \in X$ such that $\operatorname{pr}_{\iota_{n}} x=x_{n}$ for all $n$, we have $x \in Z^{\prime} \cap X \subset Z \cap X$. Hence $Z \cap X \neq \varnothing$, which completes the proof.

CoRollary 7.18. If $\left(X_{\iota}\right)_{\epsilon \in I}$ is a family of pseudocompact zeroset spaces, $X=\Pi X_{\iota}$ is pseudocompact.

Proof. We have $v X=\Pi \cup X_{\iota}=\Pi \beta X_{\iota}$ is compact; hence $v X=\beta X$.

\section{Baire sets and Baire functions.}

8.1. In this section, as an application of the preceding material, we discuss Baire sets and Baire functions. Given a zero-set space $(X, \mathscr{Z})$, one may naturally inquire about the collection $\mathfrak{B}_{1} \mathscr{Z}$ of all countable unions of zero-sets. Also the smallest collection $\mathfrak{B} \mathscr{Z}$ of subsets of $X$ which contains $\mathscr{F}$ and is closed under complementation, countable union and countable intersection suggests itself for study. The easiest way to see that $\mathfrak{B}_{1} \mathscr{Z}$ and $\mathfrak{B} \mathscr{Z}$ are zero-set structures is to refer to Hahn [4], as explained in a moment. In the case of $\mathfrak{B} \mathscr{z}$, the second easiest way is to check the axioms.

8.2. Starting again with $(X, \mathscr{L})$, we can proceed in an apparently different direction by defining $\mathfrak{B}_{1} S(X, \mathscr{Z})$ to be the ring of functions which are pointwise limits of functions in $S(X, \mathscr{Z})$. Likewise we define $\mathfrak{B S}(X, \mathscr{Z})$ to be the smallest ring of functions which contains $S(X, \mathscr{Z})$ and is closed under pointwise convergence. According to $[4,31.4 .3]$ a function belongs to $\mathfrak{B}_{1} S(X, \mathscr{Z})$ if and only if the inverse image of every closed set of $\boldsymbol{R}$ belongs to $\mathfrak{B}_{1} \mathscr{Z}$; in short, $\mathfrak{B}_{1} \mathscr{Z}$ is a zero-set structure and $S\left(X, \mathfrak{B}_{1} \mathscr{L}\right)=\mathfrak{B}_{1} S(X, \mathscr{L})$. (That $\mathfrak{B}_{1} S(X, \mathscr{Z})$ is uniformly closed is [4, 31.4.1].) Similarly according to [4, 34.3.41], a function belongs to $\mathfrak{B S}(X, \mathscr{Z})$ if and only if the inverse image of every closed set of $\boldsymbol{R}$ belongs to $\mathfrak{B} \mathscr{F}$; in short, $\mathfrak{B} \mathscr{Z}$ is a zero-set structure and $S(X, \mathfrak{B} \mathscr{Z})=\mathfrak{B} S(X, \mathscr{Z})$. We call the sets in $\mathfrak{B} \mathscr{Z}$ the Baire sets of $(X, \mathscr{Z})$ and the functions in $\mathfrak{B} S(X, \mathscr{Z})$ the Baire functions of $(X, \mathscr{Z})$; those sets and functions in $\mathfrak{B}_{1} \mathscr{Z}$ and $\mathfrak{B}_{1} S(X, \mathscr{Z})$ are specified by the phrase "of the first class."

8.3. We recall for use below that every Baire set is a union of zero-sets; i.e., each set in $\mathfrak{B} \mathscr{Z}$ is a union of sets in $\mathscr{Z}$. This 
remark is verified by letting $\mathscr{A}$ be the set of all those $A \in \mathfrak{B} \mathscr{Z}$ such that both $A$ and $X \backslash A$ are unions of sets in $\mathscr{Z}$. Then $\mathscr{A} \supset \mathscr{Z}$ and is closed under countable union, countable intersection and complementation; hence $\mathscr{A}=\mathfrak{B} \mathscr{\not}$.

8.4. The sufficiency part of the following theorem was proved for a compact space $X$ by Lorch [5, Th. 15].

THEOREM. In order that $(X, \mathfrak{B} \mathscr{Z})$ be realcompact it is necessary and sufficient that $(X, \mathscr{Z})$ be realcompact.

Proof. Sufficiency. Let $P$ be a multiplicative linear functional on $\mathfrak{B} S(X, \mathscr{Z})=S(X, \mathfrak{B} \mathscr{Z})$. The restriction $Q$ of $P$ to $S(X, \mathscr{Z})$ is a multiplicative linear functional, and hence, since $X$ is realcompact, there is a $q \in X$ with $Q f=f(q)$ for all $f \in S(X, \mathscr{Z})$. To show $P f=$ $f(q)$ for all $f \in S(X, \mathfrak{B} \mathscr{Z})$, we assume the contrary. Then the linear functionals $P$ and $f \rightarrow f(q)$ are distinct, and hence there is an $f_{0} \in S(X, \mathfrak{B} \not{Z})$ for which $P f_{0}=0$ but $f_{0}(q) \neq 0$. Consider the set $A=\left\{x \in X \mid f_{0}(x)=f_{0}(q)\right\} \in \mathfrak{B} \mathscr{Z}$. This set contains a set $Z \in \mathscr{Z}$ with $q \in Z$. Choose a $g \in S(X$, 芒 $)$ with $Z g=Z$ and set $h=f_{0}^{2}+g^{2}$. Note that $h$ vanishes nowhere on $X$, hence $h$ is a unit of $S(X, \mathfrak{B} \mathscr{Z})$, but

$$
P h=\left(P f_{0}\right)^{2}+(Q g)^{2}=0+[g(q)]^{2}=0 .
$$

This contradiction establishes $P f=f(q)$ for all $f \in S(X, \mathfrak{B} \mathscr{F})$, and thus completes the proof that $(X, \mathfrak{B} \mathscr{Z})$ is realcompact.

Necessity. Suppose $(X, \mathscr{Z})$ is not realcompact; we show that $(X, \mathfrak{B} \mathscr{z})$ is not realcompact. We know there is a $p \in v(X, \mathscr{Z}) \backslash X . p$ is the limit of a real $z$-ultrafilter $\mathscr{A}^{p}$ with respect to $\mathscr{Z}$. For each $f \in S(X, \mathscr{L}), f\left(\mathscr{A}^{p}\right)$ converges to $f^{v}(p)$, where $f^{v}$ is the continuous extension of $f$ to $v(X, \mathscr{Z})$. Since $p \notin X$, for each $x \in X$ there is an $f \in S(X, \mathscr{\gamma})$ such that $f\left(\mathscr{A}^{p}\right)$ does not converge to $f(x)$. We show next that $f\left(\mathscr{A}^{p}\right)$ is a convergent filter base on $\boldsymbol{R}$ for each $f \in \mathfrak{B} S(X$, $\mathscr{Z})=S(X, \mathfrak{B} \mathscr{\varkappa})$. Let $\mathscr{l l}$ be the set of $f \in \mathfrak{B} S(X, \mathscr{Z})$ for which $f\left(\mathscr{X}^{p}\right)$ is convergent; since $S(X, \mathscr{R}) \subset \mathscr{C}$, it is enough to show that $\mathscr{C}$ is closed under pointwise convergence. Let $f_{n} \rightarrow f$ pointwise with $f_{n} \in \mathscr{l l}$ for all $n$. Let $\delta>0$ be given. If we show $f\left(\mathscr{A}^{p}\right)$ contains a set of diameter $\leqq \delta$, we shall know $f\left(\mathscr{A}^{p}\right)$ converges and hence $f \in \mathscr{l l}$. For each $n$, choose $A_{n} \in \mathscr{A}^{p}$ such that diam $f_{n}\left(A_{n}\right)<\delta / 2$. Let

$$
A=\bigcap_{n=1}^{\infty} A_{n}
$$

since $\mathscr{A}^{p}$ is real, $A \in \mathscr{A}^{p}$. Let $x$ and $y \in A$. Choosing $n$ such that 
$\left|f_{n}(x)-f(x)\right|<\delta / 4$ and $\left|f_{n}(y)-f(y)\right|<\delta / 4$ and noting $x$ and $y \in A \subset A_{n}$, we have $|f(x)-f(y)|<\delta$. Thus diam $A \leqq \delta$; which completes the proof that $f\left(\mathscr{X}^{p}\right)$ converges for all $f \in S(X, \mathfrak{B} \mathscr{Z})$. $f \rightarrow \lim f\left(\mathscr{A}^{p}\right)$ is a multiplicative linear functional on $S(X, \mathfrak{B} \mathscr{Z})$ and, as we have already noted, there is no $x \in X$ with $\lim f\left(\mathscr{A}^{p}\right)=f(x)$ for all $f \in S(X, \mathscr{Z}) \subset S(X, \mathfrak{B} \not{Z})$. Thus $(X, \mathfrak{B} \mathscr{Z})$ is not realcompact.

COROLLARY 8.5. In order that $\left(X, \mathfrak{B}_{1} \mathscr{Z}\right)$ be realcompact it is nscessary and sufficient that $(X, \mathscr{Z})$ be realcompact.

Proof. Apply the theorem twice noting $\mathfrak{B}\left(\mathfrak{B}_{1} \mathscr{Z}^{\prime}\right)=\mathfrak{B} \mathscr{Z}$.

THEOREM 8.6. (X, $\left.\mathfrak{B}_{1} \mathscr{Z}\right)$ is never pseudocompact, unless $X$ contains only finitely many points.

Proof. We first show that for each infinite subset $B$ of $X$, we can find a $Z \in \mathscr{Z}$ such that $B \cap Z$ is infinite and $B \cap(X \backslash Z)$ is nonempty. Given such a $B$, we begin by choosing $x$ and $y \in B$ and $f \in S(X, \mathscr{Z})$ with $f(x)=1$ and $f(y)=-1$. Then $x \in B \cap Z f^{-}$and $y \in B \cap \boldsymbol{Z} f^{+}$. Since $\boldsymbol{Z} f^{+} \cup \boldsymbol{Z} f^{-}=\boldsymbol{X}$, one of the sets $\boldsymbol{Z} f^{+}$and $\boldsymbol{Z} f^{-}$has an infinite intersection with $B$; let $Z$ be this one, which is as required.

Now we construct a sequence $Z_{0}, Z_{1}, Z_{2}, \cdots$ of sets in $\not \subset$ be setting $Z_{0}=X$ and then defining the other sets in turn. For each $n \geqq 1$, we use the preceding paragraph to choose $Z_{n}$ such that

$$
Z_{0} \cap \cdots \cap Z_{n-1} \cap Z_{n}
$$

is infinite and

$$
Z_{0} \cap \cdots \cap Z_{n-1} \cap\left(X \backslash Z_{n}\right)
$$

is nonempty. Setting

$$
A_{n}=Z_{0} \cap \cdots \cap Z_{n-1} \cap\left(X \backslash Z_{n}\right)
$$

for all $n \geqq 1$ and

$$
A_{0}=Z_{0} \cap Z_{1} \cap \cdots,
$$

we note that the sets $A_{n}$ are pairwise disjoint and exhaust $X$. Whenever $Z \in \mathscr{Z}$, it follows from the definition of $\mathfrak{B}_{1} \mathscr{Z}$ that both $Z \in \mathfrak{B}_{1} \mathscr{Z}$ and $X \backslash Z \in \mathfrak{B}_{1} \mathscr{Z}$. Thus since $A_{0}$ and each $Z_{i} \in \mathscr{Z}, X \backslash A_{n} \in \mathfrak{B}_{1} \mathscr{Z}$ for all $n \geqq 0$. Thus defining $f: X \rightarrow \boldsymbol{R}$ to be $n$ on $A_{n}$, we have found an unbounded $f \in S\left(X, \mathfrak{B}_{1} \not{Z}\right)$. The result now follows by Theorem 7.6.

Corollary 8.7. (X, $\mathfrak{B} \mathscr{Z})$ is never pseudocompact, unless $X$ is finite. 
Proof. $\mathfrak{B} \mathscr{Z}=\mathfrak{B}_{1}(\mathfrak{B} \mathscr{Z})$.

\section{REFERENCES}

1. Eduard Čech, Topological Spaces, Wiley, London, 1966.

2. Leonard Gillman and Meyer Jerison, Rings of Continuous Functions, Van Nostrand, Princeton, 1960.

3. Hugh Gordon, Compactifications defined by means of generalized ultrafilters, Ann. Math. Pura Appl. Series IV 86 (1970), 15-24.

4. Hans Hahn, Reelle Funktionen, reprint, Chelsea, New York, 1948.

5. Edgar R. Lorch, Compactification, Baire functions, and Daniell integration, Acta Sci. Math. (Szeged) 24 (1963), 204-218.

Received June 16, 1969. Research partially supported by National Science Foundation grant GU-3171.

State University of New York at Albany 



\title{
PACIFIC JOURNAL OF MATHEMATICS
}

\author{
EDITORS
}

H. SAMELSON

Stanford University

Stanford, California 94305

C. R. Новву

University of Washington

Seattle, Washington 98105
J. DUGUNDJI

Department of Mathematics

University of Southern California

Los Angeles, California 90007

RICHARD ARENS

University of California

Los Angeles, California 90024

\section{ASSOCIATE EDITORS}

E. F. BECKENBACH

B. H. NeUManN

F. WOLF

K. YosHidA

\section{SUPPORTING INSTITUTIONS}

UNIVERSITY OF BRITISH COLUMBIA CALIFORNIA INSTITUTE OF TECHNOLOGY UNIVERSITY OF CALIFORNIA MONTANA STATE UNIVERSITY UNIVERSITY OF NEVADA NEW MEXICO STATE UNIVERSITY OREGON STATE UNIVERSITY UNIVERSITY OF OREGON OSAKA UNIVERSITY UNIVERSITY OF SOUTHERN CALIFORNIA
STANFORD UNIVERSITY UNIVERSITY OF TOKYO UNIVERSITY OF UTAH WASHINGTON STATE UNIVERSITY UNIVERSITY OF WASHINGTON

AMERICAN MATHEMATICAL SOCIETY CHEVRON RESEARCH CORPORATION TRW SYSTEMS

NAVAL WEAPONS CENTER 


\section{Pacific Journal of Mathematics}

\section{Vol. 36, No. $1 \quad$ November, 1971}

Norman Larrabee Alling, Analytic and harmonic obstruction on

nonorientable Klein surfaces ............................ 1

Shimshon A. Amitsur, Embeddings in matrix rings .............. 21

William Louis Armacost, The Frobenius reciprocity theorem and essentially bounded induced representations ....................... 31

Kenneth Paul Baclawski and Kenneth Kapp, Topisms and induced

non-associative systems ............................ 45

George M. Bergman, The index of a group in a semigroup ............ 55

Simeon M. Berman, Excursions above high levels for stationary Gaussian

processes....................................... 63

Peter Southcott Bullen, A criterion for $n$-convexity .............. 81

W. Homer Carlisle, III, Residual finiteness of finitely generated commutative

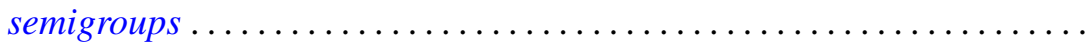

Roger Clement Crocker, On the sum of a prime and of two powers of

two ............................................ 103

David Eisenbud and Phillip Alan Griffith, The structure of serial rings . . . 109

Timothy V. Fossum, Characters and orthogonality in Frobenius

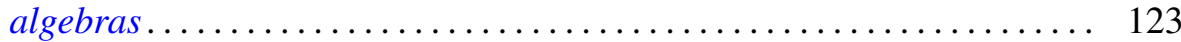

Hugh Gordon, Rings of functions determined by zero-sets . .......... 133

William Ray Hare, Jr. and John Willis Kenelly, Characterizations of Radon partitions...

Philip Hartman, On third order, nonlinear, singular boundary value

problems

David Michael Henry, Conditions for countable bases in spaces of

countable and point-countable type ..

James R. Holub, Hilbertian operators and reflexive tensor products ...

Robert P. Kaufman, Lacunary series and probability ..... . .

195

Erwin Kreyszig, On Bergman operators for partial differential equations in

two variables ................................

Chin-pi Lu, Local rings with noetherian filtrations . .

Louis Edward Narens, A nonstandard proof of the Jordan curve theorem...

S. P. Philipp, Victor Lenard Shapiro and William Hall Sills, The Abel summability of conjugate multiple Fourier-Stieltjes integrals. .

Joseph Earl Valentine and Stanley G. Wayment, Wilson angles in linear normed spaces

Hoyt D. Warner, Finite primes in simple algebras ...

Horst Günter Zimmer, An elementary proof of the Riemann hypothesis for an elliptic curve over a finite field... 